MADRS scores are reported here, as they are more sensitive to change. Response to ECT is defined as MADRS score under 11 at completion of the course.

There were 9 men and 14 women, with mean age of 58.9 years (range 24-76). Nine had a psychosis, and 14 melancholia. There were 13 ECT responders and 10 non-responders. They were a severely ill group with a mean MADRS score on entry of $\mathbf{4 3 . 2}$ (range 28-52). For nine patients, the MADRS score more than halved after two applications of ECT. The reduction of score for the whole sample after two applications was highly significant (paired $t$-test: $t=4.406, P<0.001$ ).

Of the 13 responders, 8 had their score at least halved after two applications of ECT, as did five $(56 \%)$ with psychosis, but only four $(29 \%)$ with melancholia and one (10\%) non-responder. The change of MADRS score after two ECT applications was significant for each group except melancholics (paired $t$-test: responders, $t=3.509$, $P<0.005$; non-responders, $t=2.67 ; P<0.025$; psychotics, $t=2.98, P<0.01$ ). Of the total reduction in MADRS scores at completion of ECT, $46 \%$ occurred after two treatments (45\% responders, $46 \%$ non-responders, $56 \%$ psychosis, $36 \%$ melancholia). The mean length of ECT course was 8.7 treatments (range 5-16).

These results are similar to those of Rodger et al, and support their contention that a substantial benefit from ECT is realised after early treatments. This sample was more depressed (mean HRSD score before $\mathrm{ECT}=33$, s.e.m. $=1.6$ ), assessments were performed after two, not three treatments, and subjects were not drug free. These factors may contribute to the larger effects found by Rodger et al.

Why do some and not others respond early? Does response time distinguish different subtypes of disorder? What are the implications for the biology of depression? As the most potent and consistent antidepressant treatment for severe depression, understanding the mechanism of action and cerebral effects of ECT is of great significance. Clearly, we have much to learn about ECT, but the discrete, definable nature of this treatment lends itself to study. Its secrets may teach us much that we need to know about affective disorders.

American Psychintric Association (1980) Diagnostic and Statistical Manual of Mental Disorders (3rd edn) (DSM-III). Washington, DC: APA.

Anderson, D. N., Abou-Saleh, M. T., Collnns, J., et al (1992) Pterin metabolism in depression: an extension of the amine hypothesis and possible marker of response to ECT. Psychological Medicine, 22, 863-869.
Hammton, M. (1969) Standardised assessment of depressive symptoms. Psychiatry. Neurology and Neurosurgery, 72, 201205.

Montgomerry, S. A. \& Asberg, M. (1979) A new depression rating scale designed to be sensitive to change. British Journal of Psychiatry. 134, 382-389.

DAVID N. ANDERSON

Department of Psychogeriatrics

Mossley Hill Hospital

Liverpool L18 8BU

\section{Electroconvulsive therapy and brain damage}

SIR: Some critics still believe that electroconvulsive therapy (ECT) inevitably leads to brain damage and intellectual impairment (Breggin, 1993), although prospective quantitative magnetic resonance imaging has not found any structural changes in the cerebral hemispheres after two or three courses of ECT (Scott et al, 1991). There has never been an adequate study of the long-term intellectual effects of ECT because prospective studies have been limited to the assessment of patients before and after only one course of ECT (maximum 12 treatments). We report a prospective assessment of the intellectual effects of 125 treatments given to a patient over seven years; a preliminary report has already appeared in the BJP (December 1991, 159, 867-870).

Case report. A 67-year-old catering assistant suffered a recurrence of a depressive illness, and required admission. She was agitated, anxious and depressed, expressing delusions of guilt, and had tried to kill herself. She had suffered five previous episodes of depressive illness that had not responded to antidepressant drugs, but had to ECT. The index episode responded preferentially to bilateral ECT, but relapse was not prevented by antidepressant with neuroleptic drugs in doses she would tolerate. Two years later she agreed to fortnightly bilateral ECT as continuation treatment. This was successful, and she was discharged to a private nursing home. She continued to receive ECT as an out-patient and remained well; the frequency of ECT was reduced to once every three weeks two and a half years later. The baseline intellectual assessments were carried out by an independent clinical psychologist a year before the patient's admission, when she was well and had not received ECT in seven years. The later assessments were carried out by a psychiatrist (AGH) who knew the patient was being treated by ECT, but was unaware of treatment details. The patient was assessed 10 days after the last ECT and was free from depressive symptoms at each visit. Psychotropic drug treatment was standard (10 mg trifluoperazine).

At the baseline assessment she was fully orientated and her mental abilities according to the Clifton Assessment Procedure for the Elderly (CAPE; Pattie \& Guillard, 1979) placed her in the category of 'independent elderly' (Table 1). This did not change after 125 ECT treatments. Her 
CORRESPONDENCE

Table 1

\begin{tabular}{lccc}
\hline & Baseline & After 100 ECT & After 125 ECT \\
\hline Raw scores on CAPE & & & \\
information orientation (max. 12) & 12 & 12 & 12 \\
mental ability (max. 11) & 11 & 11 & 11 \\
psychomotor skill (max. 12) & 11 & 11 & 11 \\
Premorbid IQ & 103 & 105 & 108 \\
Contemporaneous 10 & 99 & 100 & 100 \\
\hline
\end{tabular}

contemporaneous IQ, estimated using the Mill Hill Vocabulary Scale (Raven et al, 1982), was almost exactly the average at the initial onset and did not differ from her predicted premorbid IQ (National Adult Reading Test; Nelson, 1982): this did not change.

A single case study cannot resolve the continuing debate about ECT and brain damage, but the present case is unique in the literature and shows clearly that a prolonged course of bilateral ECT had no effect upon the intellectual abilities assessed. Long-term prospective studies that cover a wider range of intellectual functions are still required.

Breggn, P. (1993) Toxic Psychiatry: Drugs and Electroconvulsive Therapy - The Truth and Better Alternatives. London: Fontana.

Nelson, H. E. (1982) National Adult Reading Test: Test Manual. Windsor: NFER

Pattie, A. H. \& Guillard, C. J. (1979) The Clifton Assessment Procedure for the Elderly. Seven Oaks: Hodder and Stoughton. Raven, J. C., Raven, J. \& Court, J. H. (1982) The Mill Hill Vocabulary Scale, Form 2 Senior. London: H.K. Lewis.

Scott, A. I. F., TURNBUlL, L. W., Blane, A., et al (1991)

Electroconvulsive therapy and brain damage. Lancet, 338, 284.

A. G. HaY

Royal Edinburgh Hospital

A. I. F. SCOTT

Edinburgh ENI0 5HF

\section{Volunteers to help Bosnian refugees}

SIR: We are hoping to set up a project offering psychiatric and counselling support for the Bosnian refugees in the London area. The refugees have almost without exception been subjected to appalling psychological and physical trauma, including torture, rape, and detention in concentration camps. Many of the survivors suffer from posttraumatic stress disorder (PTSD), and a small proportion are affected by major psychiatric illness, including severe depression and psychotic reactions. There is a lack of appropriate and accessible health and social services for refugees and asylum-seekers and this project is a collaboration between the Traumatic Stress Clinic (Camden and Islington
Community Trust), which is a national referral centre specialising in therapeutic interventions for people with PTSD, and psychiatrists at Guy's Hospital with an interest in transcultural psychiatry and a history of service development for the Indo-Chinese refugee community.

The Bosnian project is coordinated by the Jewish Council for Community Relations and is working in association with the Department of Child Psychiatry at the Royal Free Hospital, the Marborough Family Service, the Medical Foundation for the Victims of Torture, the Refugee Council, and the Bosnian Embassy. The bulk of the therapeutic activity will be carried out by bi-cultural workers using cognitive models of intervention developed and evaluated within the Traumatic Stress Clinic using methods successfully used within a specialist service for survivors of organised violence in Turkey (Yuksel, 1990). The bi-cultural workers will screen people who have evidence of major psychiatric disorder, who will be referred to a network of mental health practitioners. This team of practitioners will be able to carry out domiciliary and community assessments, with the help of interpreters.

Psychiatrists, community psychiatric nurses and other mental health professionals who wish to participate in this project on a voluntary basis should contact Dr Edie Friedman on 081455-0896. We are also looking for charitable funding.

Yuksel S. (1990) Post-traumatic cognitive behaviour therapy. Proceedings of the Second European Conference on Traumatic Stress, p. 58. Netherlands: Noordwijkerhort.

STUART TURNER

Camden \& Islington CHS (NHS) Trust

112 Hampstead Road

London NWI $2 L T$

Department of Psychiatry

MAURICE LiPSEDGe

Guy's Hospital,

London 